\title{
notes
}

\section{Effets des rayonnements ionisants sur le développement in utero}

\author{
J. LALLEMAND* \\ (Manuscrit reçu le 27 septembre 1983)
}

\section{RÉSUME}

\begin{abstract}
Dans cet article sont abordés successivement, après quelques rappels embryologiques et méthodologiques, les principaux effets tératogènes liés à une exposition aux rayonnements ionisants, à savoir l'effet létal des rayonnements ionisants, les malformations radioinduites, les maldéveloppements et les cancers. Bien que la susceptibilité de l'embryon et du fœtus à l'irradiation semble exister durant toute la période de la gestation, les travaux récents, sur lesquels nous avons cru bon d'insister, nous apprennent que l'effet dommageable principal est le retard mental.

En conclusion, cette étude débouche sur des considérations pratiques de radioprotection dans le cadre des examens radiographiques des femmes enceintes.
\end{abstract}

\section{ABSTRACT}

Following a reminder of embryology and methodology, a review is made of the main teratogenic effects related to radiation exposure, i.e. lethal effects, radioinduced malformations, maldevelopment and cancers. The sensitivity of the embryo and foetus to radiation seems to last during the whole gestation. Howewer, the latest investigations indicate that the main damage is mental retardation.

This review concludes on practical considerations of radiation protection in the field of radiographic examinations of pregnant women.

\section{INTRODUCTION}

II est communément admis que l'embryon et le fœtus humain sont très sensibles aux effets de l'irradiation. A l'instar de nombreux toxiques physiques ou chimiques, cette sensibilité aux rayonnements ionisants connaît des variations quantitatives et qualitatives en fonction du stade du développement.

\footnotetext{
* EdF, Comité de radioprotection, 39, rue de Washington, 75008 Paris.
} 
Mise à part la cancérogénèse radioinduite, les dommages les plus graves qui puissent advenir à la suite d'une irradiation prénatale, dans l'espèce humaine, sont les retards mentaux [17-19]. Les périodes de sensibilité maximale du produit de conception sont actuellement remises en question. En effet, une généralisation trop hâtive des expérimentations animales a fait oublier la spécificité du développement humain. $\mathrm{Ce}$ sont les progrès récents en embryologie et l'essor de la biologie du cerveau humain qui permettent, à présent, de préciser la notion de période sensible.

\section{GENERALITES}

Avant d'aborder l'étude des effets tératogènes à proprement parler, il convient de rappeler brièvement quelques données d'embryologie fondamentale et de méthodologie.

\section{Données embryologiques}

Durant la période de pré-implantation qui a une durée voisine chez tous les mammifères, les cellules sont indifférenciées et totipotentes. Ceci explique qu'une irradiation brève à forte dose est susceptible de tuer l'embryon, mais incapable d'induire des malformations. Des individus parfaitement normaux peuvent se développer à partir d'une seule cellule de la morula.

A ce stade précoce du développement, les effets d'une irradiation sont donc du type "tout ou rien" [37]. Ils peuvent aboutir à la mort de l'embryon, qui passe le plus souvent inaperçue, ou permettre une croissance et une survie post-natale normales.

Après l'implantation de l'œuf $\left(6^{e}-9^{e}\right.$ jour dans l'espèce humaine), se situe la période de morphogénèse active, durant laquelle se mettent en place les ébauches des différents tissus et organes. II s'agit là de la période tératogène par excellence : toute interférence avec la différenciation clonale des ébauches des différents organes va se traduire par la survenue de malformations.

II importe donc de souligner d'emblée que, malgré sa courte durée (60 jours chez l'homme), la période embryonnaire est fondamentale, puisque l'embryon acquiert sa forme presque définitive (morphogénèse) et édifie ses principales ébauches organiques (organogénèse). Ainsi, chaque tissu passe par des périodes de radiosensibilité maximale qui correspondent à la phase de différenciation et d'organisation de ce tissu ; une mort cellulaire, à un moment où les cellules différenciées sont encore peu nombreuses, peut entraîner un arrêt de développement de l'organe ou d'une de ses parties et donc une anomalie majeure. En tenant compte de la chronologie du développement embryonnaire, les phases de risque maximal sont les mêmes pour toutes les espèces de mammifères.

Pendant la longue période fœtale qui lui succède, les organes ne subiront pratiquement plus que des phénomènes de maturation à l'échelle histologique (histogénèse). 


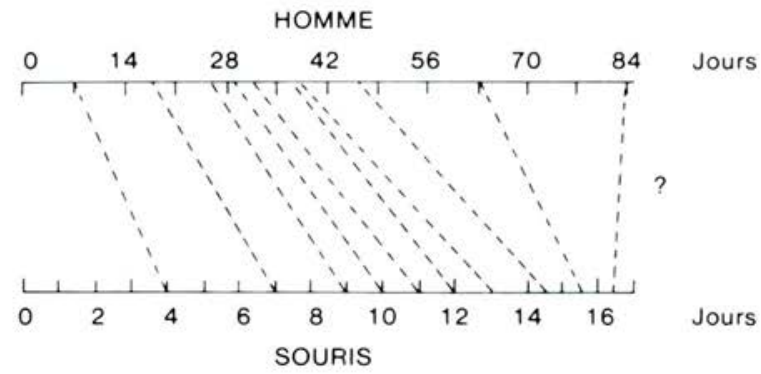

Fig. 1. - Correspondance entre les étapes du développement des embryons humains et de souris [40].

TABLEAU I

Principales périodes du développement (en jours) [37]

\begin{tabular}{|c|c|c|c|}
\hline & Pré-implantation & Organogénèse & Période fœtale \\
\hline Souris ............. & 0.5 & $6-12$ & 13- 16,5 \\
\hline Rat $\ldots \ldots \ldots \ldots$ & $0-7$ & $8-15$ & $16 \cdot 21,5$ \\
\hline Homme ..... & $0-8$ & $9-60$ & $\begin{array}{c}60-270 \\
\text { (du } 60 \text { au } 110^{\circ} \text { jour } \\
\text { prolifération } \\
\text { neuroblastes) }\end{array}$ \\
\hline \multirow[t]{4}{*}{$\begin{array}{l}\text { Effets des } \\
\text { irradiations }\end{array}$} & - Mort intra-utérine & $\begin{array}{l}\text { - Mort intra-utérine } \\
\text { (début de l'organogé- } \\
\text { nèse) }\end{array}$ & $\begin{array}{l}\text { - Malformations } \\
\text { nerveuses et } \\
\text { retard mental } \\
\text { (du } 60 \text { au } 110^{\text {e jour) }}\end{array}$ \\
\hline & $\begin{array}{l}\text { Croissance et survie } \\
\text { post-natales normales }\end{array}$ & $\begin{array}{l}\text { - Malformations et } \\
\text { maldéveloppements } \\
\text { du SNC* }\end{array}$ & - Défauts de croissance \\
\hline & & $\begin{array}{l}\text { - Mort néo-natale et } \\
\text { post-natale }\end{array}$ & - Fragilité post-natale \\
\hline & & & - Cancers de l'enfance \\
\hline
\end{tabular}

* $\mathrm{SNC}=$ système nerveux central.

II est très intéressant de souligner une donnée d'embryologie comparée, qui explique peut-être la moindre radiosensibilité de l'embryon humain. Le développement initial est beaucoup plus lent chez l'homme que dans bien d'autres espèces. Ce taux de division cellulaire plus faible explique la moindre radiosensibilité de l'embryon humain; en effet, plus le taux de division, donc l'activité mitotique, est faible, moins il y a 
de cellules en phase radiosensible. Par ailleurs, plus la durée d'un stade déterminé du développement embryonnaire est longue, plus la récupération de croissance est efficace.

II est intéressant, à présent, d'aborder brièvement les données nouvelles concernant le développement du cerveau.

Durant les six premières semaines, le développement de l'ectoblaste aboutit à la mise en place du cerveau primitif. La vésicule télencéphalique donne naissance aux hémisphères cérébraux, reconnaissables chez l'homme à la fin de la septième semaine p.c. (post-conception). A partir de ce moment, cet organe primordial croît et se développe, les hémisphères cérébraux poursuivent leur croissance et leur différenciation durant toute la vie fœtale et aussi durant les premières années de la vie post-natale. La période sensible ne se limite donc pas à l'embryogénèse.

En même temps que s'opère la mise en place des structures anatomiques, les neuroblastes, cellules nobles précurseurs des neurones, se divisent. Cette division cellulaire est exponentielle, comme l'atteste l'augmentation du contenu en $A D N$, mais elle est limitée dans le temps à une période qui s'étend de la $8^{e}$ à la $16^{e}$ semaine [18]. L'irradiation, durant cette période, entraîne essentiellement une perte de la capacité de prolifération; mais des neuroblastes ainsi agressés peuvent également mourir en interphase.

La période sensible entre toutes est donc la période de prolifération rapide des neuroblastes. Seule, une déplétion cellulaire modérée peut être compensée par une augmentation de l'activité mitotique des neuroblastes. Mais, la période sensible pour la mise en place des structures cérébrales ne se limite pas à cette seule période de prolifération rapide des neuroblastes. En effet :

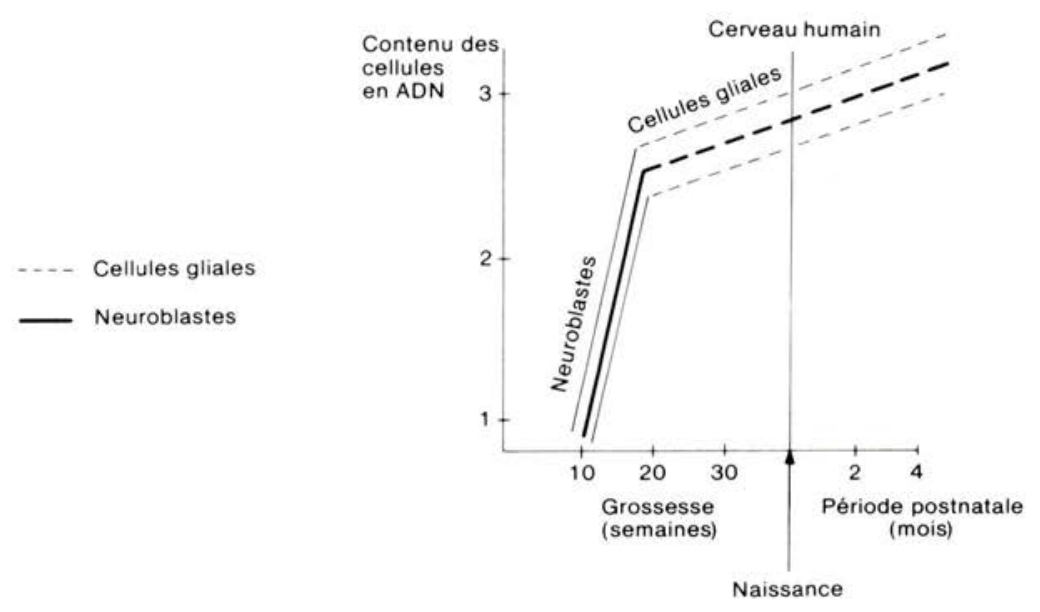

Fig. 2. - Evolution du contenu des cellules en ADN en fonction du temps [7]. 
- un nombre très réduit de neuroblastes précurseurs de microneurones se divisent dans le cervelet et dans l'hippocampe durant la période périnatale chez l'homme [7 - 8], ce contingent cellulaire est donc particulièrement vulnérable à l'irradiation survenant en fin de grossesse ;

- par ailleurs, on assiste, à partir de la $17^{\mathrm{e}}$ semaine p.c. et jusqu'à plusieurs mois après la naissance, à une deuxième période de multiplication cellulaire; elle concerne les précurseurs des cellules gliales: les spongioblastes (fig. 2).

L'importance de cette prolifération gliale peut être soulignée en évoquant deux fonctions fondamentales de ce tissu de soutien :

- les fibrilles gliales guident la migration des neurones, dont très peu naissent au site où ils vont acquérir une activité fonctionnelle ;

- par ailleurs, la synthèse de la myéline dépend, elle aussi, des cellules gliales.

Donc :

- la nature du facteur responsable d'un effet tératogène est moins importante à considérer que le moment précis de la gestation, où il intervient ;

- un même agent tératogène peut donner des malformations différentes, s'il est administré à des stades différents de la morphogénèse ;

- enfin, étant donné la simultanéité du développement de plusieurs ébauches, un même agent peut engendrer l'association de plusieurs malformations.

Pour ce qui concerne les tissus autres que le système nerveux, il importe de distinguer de façon nette les périodes embryonnaire et fœtale :

- pendant la période d'embryogénèse, chaque tissu passe par une période de radiosensibilité maximale correspondant à la phase de différenciation et d'organisation de ce tissu; au moment de la radiosensibilité maximale, des doses aussi faibles que 5 cGy $^{1}$ peuvent entraîner des malformations notables chez l'animal, mais la radiosensibilité diminue avant et après cette période critique ;

- pendant la période fœtale, la fréquence et la gravité des malformations diminue, et les effets tératogènes deviennent moins importants, peut être parce que le nombre de cellules dans chacun des tissus est suffisamment grand pour qu'il soit peu probable d'en léser un nombre suffisant pour créer une malformation grave.

\section{Méthodologie}

Les effets tératogènes des rayonnements ionisants sont étudiés par l'expérimentation animale et l'étude épidémiologique chez l'homme.

\section{Expérimentation animale}

Les études expérimentales conduites essentiellement sur de petits mammifères (souris), mais également, plus récemment, sur le chien

1. Bien que le cGy ne soit pas une unité habituellement utilisée, nous l'emploierons dans cet article, dans le but de réaliser une transition plus facile entre l'ancien et le nouveau système d'unités. En effet : $1 \mathrm{cGy}=1 \mathrm{rad}$. 
beagle pour l'effet cancérogène, permettent de maîtriser tous les paramètres susceptibles de modifier l'effet (dose, débit de dose, âge gestationnel,...) et de définir de façon précise le groupe témoin. Des techniques nouvelles ont été mises au point afin d'étudier des altérations fines. La culture d'embryons in vitro durant la pré-implantation, par exemple, permet d'étudier les phénomènes radiobiologiques généraux et les relations dose-effet dans un système biologique très radiosensible et biologiquement bien défini.

Cependant, l'extrapolation de ces données à l'homme pose de nombreux problèmes :

- L'expérimentation animale a montré la plus grande résistance des hétérozygotes à l'effet tératogène et létal d'une irradiation in utero par rapport aux homozygotes. Or, comme les expérimentations sont, le plus souvent, effectuées sur des animaux de souche pure, une prudence particulière s'impose avant d'extrapoler à l'homme [29, 33, 35].

- Il semblerait que la radiosensibilité de l'embryon humain soit plus faible que celle des embryons des principales espèces étudiées.

- Enfin, les données expérimentales ont montré, chez l'animal que la période la plus sensible à l'action des agents tératogènes va de l'implantation à la fin de l'organogénèse. Or, comme nous l'avons développé dans le chapitre précédent, les données récentes ont fait apparaître que l'exposition aux rayonnements ionisants durant la période fœtale est susceptible d'agir sur le développement du cerveau et peut conduire à des altérations fonctionnelles sévères, qui s'expriment durant la vie postnatale. Ce type d'effet concerne sans doute essentiellement l'espèce humaine et, dans ce cas, les expérimentations animales, pour rigoureuses qu'elles soient, ne permettent pas d'extrapoler à l'homme...

\section{Epidémiologie humaine}

Les observations humaines sont assez nombreuses, mais leur intérêt est souvent limité pour des raisons très diverses; les principales sont les suivantes :

- imprécisions dosimétriques : c'est le cas à Hiroshima et à Nagasaki [1], mais ce problème est encore beaucoup plus net pour les irradiations médicales à des fins diagnostiques ;

- difficulté de constituer un groupe témoin, et c'est là l'écueil fondamental, auquel se heurtent la plupart des enquêtes épidémiologiques ;

- l'âge de l'embryon au moment de l'irradiation n'est, en général, connu que de façon très approximative, or, les effets de l'irradiation varient en fonction du moment où celle-ci a été effectuée,... ;

- enfin, d'autres facteurs [17] viennent bien souvent ajouter leurs effets à ceux des rayonnements. Parmi les plus importants, citons :

- La malnutrition: les expérimentations animales ont montré que le système nerveux central est très sensible à la malnutrition. Ceci semble être également le cas chez l'homme. Bien que l'importance relative des carences alimentaires n'ait pu être clairement précisée, on a constaté que les déficiences mentales sont plus fréquentes dans les populations sous-alimentées. L'enquête humaine la plus importante dont nous disposons, à savoir celle qui concerne les survivants d'Hiroshima 
et de Nagasaki, est peut-être biaisée par ce facteur : en effet, le rationnement alimentaire de 1945 peut être incriminé dans le nombre anormalement élevé de retards mentaux sévères déplorés dans la population japonaise non irradiée.

Les autres maladies : 7 cas de retards mentaux sévères constatés parmi les survivants japonais relevaient certainement d'autres causes. II s'agissait d'encéphalite B, de maladie de Von Recklinghausen...

Tous les autres agents tératogènes de l'environnement: médicaments, tabac, toxiques chimiques, etc.

Pour toutes ces raisons, et malgré les différences de sensibilité entre l'animal et l'homme, les données animales restent une base indispensable pour l'estimation des effets tératogènes des rayonnements ionisants.

\section{EFFET LETAL DES RAYONNEMENTS IONISANTS}

\section{Données expérimentales}

Les expériences ont été menées essentiellement sur des souris.

La radiosensibilité de l'embryon et du fœtus varie au cours du développement :

- Avant l'implantation, la radiosensibilité est très grande et la dose létale 50 (DL 50) est de l'ordre de 50 à 60 cGy. Comme l'ont montré les travaux de RUGH chez la souris [28], la valeur de la DL 50 avant nidation est d'autant plus basse que l'irradiation est intervenue de façon plus précoce. Cet auteur a obtenu la mort de produits de conception avec des doses de 10 cGy délivrées très précocement, à savoir quelques heures après la fertilisation.

- Après l'implantation, la radiosensibilité est élevée durant les premiers stades de l'organogénèse, pour diminuer ultérieurement.

Mais l'effet létal d'une irradiation ne se réduit pas à la mortalité intrautérine. On observe également une mortalité néo-natale et post-natale. En effet, des doses inférieures à 1 Gy délivrées durant la phase embryonnaire n'augmentent pas la mortalité néo-natale, mais accroissent légèrement la mortalité post-natale. II s'y ajoute, pour les survivants, et pour des doses supérieures à 2 Gy, un raccourcissement de la durée de la vie.

Pendant la période fœtale, la DL 50 à 30 jours augmente progressivement avec l'âge du fœtus, pour rejoindre, à la naissance, la DL 50 des souris adultes. Pour ce qui concerne l'effet létal, le fœtus est donc, à la fin de la vie intra-utérine, aussi résistant que l'animal adulte.

\section{Données humaines}

Les données humaines sur l'effet létal de l'irradiation in utero sont relativement peu nombreuses et assez imprécises.

- En ce qui concerne les irradiations thérapeutiques, il semble qu'une irradiation de l'ordre de 3,5 à 4 Gy soit suffisante pour provoquer une fausse couche dans la majorité des cas. KALLINGER et GRANNINGER [14] 
relatent, cependant, un cas d'irradiation thérapeutique, par radium 226 , ayant entraîné une dose fœtale estimée à 6,8 Gy, sans conséquence apparente. Cette observation doit être interprétée avec beaucoup de prudence.

Pour ce qui concerne les irradiations thérapeutiques, il importe, cependant, de préciser que les doses sont fractionnées ou délivrées sur de longues périodes. Ces observations ne peuvent donc illustrer, de façon claire, la relation existant entre l'âge gestationnel au moment de l'irradiation et le dommage qui en résulte.

- Les données d'Hiroshima et de Nagasaki montrent que sur les 30 femmes enceintes qui avaient présenté un mal des rayons immédiatement après l'explosion, ce qui indique des doses élevées ( $\geqslant 1$ Gy), la mortalité intra utérine ou néo-natale a été importante, de l'ordre de $40 \%$. Aucun cas n'a été signalé chez les femmes enceintes irradiées à des doses plus faibles.

- Sur les 1300 enfants irradiés in utero, la mortalité a été supérieure à la normale pendant la première année de la vie et elle a été correlée avec la dose.

\section{MALFORMATIONS RADIOINDUITES}

Avant d'aborder leur étude, il convient de les définir et surtout de les distinguer des maldéveloppements détaillés au chapitre 5.

Nombre de malformations importantes peuvent être rattachées à un "défaut" du développement embryonnaire [4] survenant très souvent, mais non toujours, durant la période de différenciation rapide.

Cependant, l'irradiation n'entraîne pas exclusivement des lésions macroscopiques de ce type. A l'échelle microscopique, l'absorption d'énergie lèse, au hasard, un certain nombre de cellules constituant un tissu. La mort ou l'altération fonctionnelle d'un petit nombre de cellules distribuées au hasard, n'entraîne que peu souvent des malformations aussi patentes que celles précédemment décrites. Et c'est pour cette raison qu'il convient de distinguer la malformation du maldéveloppement [17]. La malformation résulte d'un défaut d'organisation de l'embryon. Le maldéveloppement, par contre, peut se manifester en l'absence de malformations au sens strict du terme. Une déplétion cellulaire au niveau cérébral est à même d'entraîner un retard mental sévère sans lésion macroscopique.

Les malformations les plus fréquemment citées dans les expérimentations animales sont les suivantes:

- malformations du système nerveux central (exencéphalies, anencéphalies, hydrocéphalies peuvent être observées pour des doses aussi faibles que 25 cGy, mais leur fréquence est très faible pour des doses inférieures à 1 Gy et atteint environ $15 \%$ pour des doses de 1,5 Gy [37]) ;

- malformations de l'œil, observées chez la souris et le rat, à partir de 25 cGy, pendant la phase critique de l'embryogénèse correspondant à la différenciation de l'œil (pour 0,5 Gy, une fréquence de $7 \%$ de malformations est observée à la phase critique) ; 
- malformations du squelette : elles ont fait l'objet d'études nombreuses, parce qu'elles sont faciles à diagnostiquer et à quantifier. Leur fréquence est très légèrement augmentée pour des doses de 5 à 20 cGy et elle croît avec la dose. NOMURA [25], signale une augmentation importante des malformations squelettiques dans les portées issues de souris gestantes traitées par 0,36 à 5 Gy de rayons $X$.

Chez l'homme, LEJEUNE et coll ont décrit une hétérochromie segmentaire de l'iris chez les enfants irradiés in utero aux $4^{e}$ et $5^{e}$ mois de grossesse. La fréquence de cette anomalie est très faible et cette observation n'a pas pu être confirmée par d'autres auteurs.

Par ailleurs, diverses enquêtes n'ont pas mis en évidence d'augmentation de la fréquence des anomalies osseuses pour des doses de l'ordre de quelques centigrays, ce qui permet d'exclure l'hypothèse d'une radiosensibilité élevée de l'embryon humain.

Les principales conclusions, qui méritent d'être soulignées, sont les suivantes :

- L'irradiation de l'embryon en période d'organogénèse peut être responsable d'une grande variété de malformations. II est possible de définir des périodes critiques avec une radiosensibilité maximale pour chaque anomalie en fonction de l'âge.

- Dans tous les cas, les malformations ont été induites durant la période de postimplantation. L'irradiation durant la période de préimplantation est inefficace, en ce qui concerne l'induction de malformations. La plupart des auteurs admettent qu'une irradiation durant les 9 premiers jours de la conception, c'est-à-dire durant la période de préimplantation, n'entraîne aucun risque tératogène pour l'enfant à naître. De ce fait, la règle des 14 jours pourrait devenir une règle des $14+9=23$ jours. Dans cette hypothèse, seuls les 5 derniers jours d'un cycle de 28 jours seraient à éviter en cas de radiodiagnostic. Certains auteurs pensent même que cette règle des 23 jours ne se justifie pas, étant donné que l'embryon humain est beaucoup moins radiosensible que l'embryon de souris, base de l'expérimentation.

- Enfin, en considérant l'ensemble des malformations observées chez l'animal, divers auteurs ont abouti à des probabilités de malformations variant entre 2 pour 1000 et 5 pour 1000 par cGy en acceptant, a priori, l'hypothèse d'une relation linéaire. II est impossible de savoir s'il existe un seuil, car la fréquence est si faible pour les petites doses que l'on se heurte au problème de la signification statistique des données observées. II ne faut pas oublier que, dans l'espèce humaine, environ 5 à 10 pour cent des nouveaux-nés sont porteurs d'une anomalie congénitale et il est impossible de distinguer les malformations radioinduites du bruit de fond, car elles n'ont aucune spécificité.

\section{MALDEVELOPPEMENTS}

Au début des années 1950, il a été établi qu'une expositon du fœtus aux rayonnements ionisants pouvait entraîner, entre autres : 
- une augmentation de la fréquence des microcéphalies ${ }^{1}$ accompagnées ou non de retard mental sévère ${ }^{2}$;

- une diminution de la croissance durant l'enfance et l'adolescence.

Ces deux points méritent d'être quelque peu détaillés.

\section{Microcéphalie et retard mental}

De toutes les données épidémiologiques concernant l'irradiation prénatale, l'enquête la plus intéressante est certainement celle qui porte sur les survivants japonais. En effet, une irradiation unique et brève a été délivrée à des embryons et fœtus de tous âges. Rétrospectivement, on a pu connaître de façon très précise l'âge du produit de conception au moment de l'irradiation, ainsi que la dose reçue (aux imprécisions dosimétriques près) [18-19].

L'étude approfondie de cette population, suivie médicalement depuis près de 40 ans, a mis en évidence le fait fondamental suivant : le seul effet manifeste d'une irradiation in utero, chez l'homme, est l'augmentation des microcéphalies et des retards mentaux sévères. En plus, il faut noter que cette augmentation est correlée avec la dose [19].

Les observations de retards mentaux (RM) sévères chez les survivants japonais ont été analysées dans un travail récent de OTAKE et SCHULL et reprises par MOLE [18-19].

Ces auteurs ont distingué deux groupes de microcéphalies pour tenir compte de la coexistence éventuelle d'un retard mental sévère. On peut donc décrire :

\section{Microcéphalies sans retard mental}

19 cas recensés : il s'agit de sujets exposés entre la $4^{\mathrm{e}}$ et la $16^{\mathrm{e}}$ semaine de gestation (fig. 3) [18-19]. 18 cas (sur 19) sont apparus après une irradiation située entre la $4^{\mathrm{e}}$ et la $14^{\mathrm{e}}$ semaine p.c., dont 13 cas irradiés avant la $10^{\circ}$ semaine p.c. et donc avant la période de multiplication des neuroblastes. Une microcéphalie peut donc être la conséquence d'une irradiation précoce.

\section{Retard mental sévère}

18 cas recensés, 15 d'entre eux sont apparus après une exposition aux rayonnements entre la $10^{e}$ et la $16^{e}$ semaine de gestation. Aucun cas n'a été décelé avant la $10^{e}$ ni après la $26^{e}$ semaine de gestation.

1. On parle de microcéphalie, lorsque le périmètre cranien est inférieur de 2 déviations standards à celui auquel on pourrait s'attendre chez les individus de cette taille, de ce sexe et à cet âge.

2. Retard mental sévère : enfant incapable de réaliser des calculs simples, d'entretenir une conversation simple, de se suffire à lui-même. 


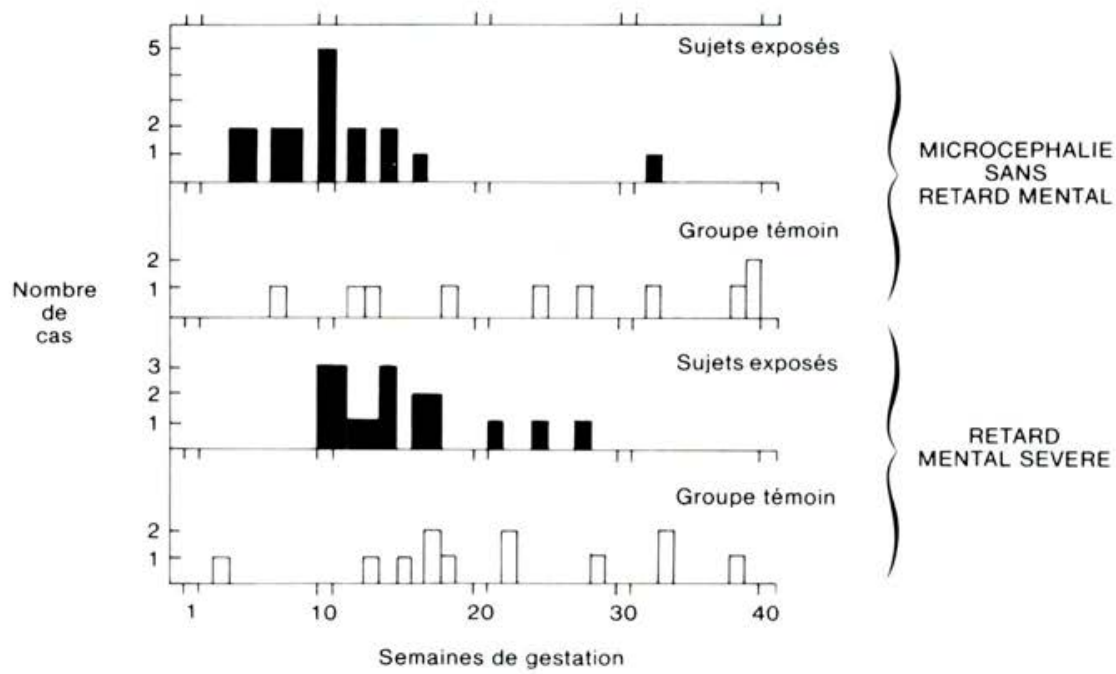

Fig. 3. - Microcéphalies et retards mentaux observés chez les survivants japonais irradiés in utero, d'après MOLE [18-19].

II ressort de ces considérations que la période s'étendant de la $8^{\text {e à }}$ la $16^{\text {e }}$ semaine de grossesse est particulièrement importante, en ce qui concerne les malformations et surtout les maldéveloppements du système nerveux central. II s'agit, en tout cas, de la période la plus sensible pour l'induction de dommages cérébraux aboutissant à un retard mental sévère.

En tenant compte de l'ensemble de ces données, on peut admettre, qu'en première approximation, chez l'homme, une irradiation de 0,5 Gy provoque un retard mental chez $5 \%$ des embryons irradiés et que cette fréquence augmente de $1 \%$ par cGy pour les doses plus élevées.

L'existence d'un seuil et l'effet éventuel de doses plus faibles restent impossibles à affirmer, en raison de problèmes méthodologiques statistiques, puisque la fréquence des retards mentaux chez les enfants non irradiés est loin d'être négligeable. Néanmoins, certains auteurs considèrent qu'on peut raisonner comme s'il n'existait pas de seuil et que la relation dose-effet restait linéaire pour des doses faibles (avec une fréquence de $1 \%$ o par cGy).

\section{Troubles de la croissance}

Chez l'animal [18] une irradiation durant les périodes embryonnaire ou fœtale provoque un retard de la croissance et du développement in utero qui se traduit par une diminution statistiquement significative du poids à la naissance et un retard de la croissance postnatale. A âge égal, les souriceaux irradiés sont plus petits et de moindre poids. Cette réduc- 
tion pondérale s'observe encore à l'âge adulte et s'accompagne d'une diminution de poids des différents viscères, en particulier, du cerveau.

Chez l'homme, ces déficits staturo-pondéraux ont été retrouvés dans les deux principales études épidémiologiques :

\section{Enfants irradiés in utero pour des raisons médicales}

Ces études confirment l'existence d'un retard de croissance chez les enfants irradiés, mais il existe une discordance entre les données fournies par les irradiations médicales et les autres, ce qui invite à la plus grande prudence dans l'interprétation de ces données [10, 12, 15, 31].

\section{Les enfants irradiés in utero à Hiroshima et Nagasaki}

Les données sont particulièrement nettes pour ceux d'entre eux qui se trouvaient à moins de $1,5 \mathrm{~km}$ de l'hypocentre, c'est-à-dire les plus irradiés :

Ces sujets ont reçu des doses supérieures à 1 Gy. A l'âge de 17 ans, on a noté chez ces sujets un déficit staturopondéral net et une réduction significative du périmètre crânien. On a noté également un retard à l'âge des premières règles et un retard d'ossification qui ne paraît cependant pas corrélé avec la dose. Par contre, on n'a pas pu mettre en évidence de relation dépendant de la dose pour 2 indices caractérisant la fertilité (fréquence des mariages sans enfant, nombre de naissances). Les modifications du sex ratio (prédominance de garçons), signalées par certains auteurs, n'ont pas été confirmées.

Enfin, de nombreuses études effectuées sur le devenir des enfants irradiés in utero à des doses très variables, et de leur descendance, études basées sur des examens cliniques répétés, n'ont révélé aucun effet significatif.

\section{LES CANCERS RADIOINDUITS}

\section{Observations animales}

Les ètudes effectuées sur de petits mammifères (essentiellement des souris) montrent que l'irradiation prénatale augmente l'incidence du cancer. Cette augmentation est d'autant plus nette que l'irradiation est intervenue tardivement (période fœtale tardive et période périnatale) [32, 38].

Afin d'éprouver la sensibilité de la période fœtale à la cancérogénèse radioinduite chez des mammifères, dont le comportement physiologique est plus proche de celui de l'homme, une expérience sur les chiens fut menée par BENJAMIN et coll [2-3]. Elle porte sur 1680 chiens beagles, des deux sexes, irradiés par les rayons gamma du cobalt 60 à différents moments de la gestation ou de la vie postnatale. Les doses délivrées ont été de 16 ou 83 cGy. Six groupes ont été constitués en fonction du moment de l'irradiation : $8^{\mathrm{e}}, 28^{\mathrm{e}}$ et $55^{\mathrm{e}}$ jours post-conception (dpc) (c'est-àdire au début, au milieu et en fin de gestation), $2^{\mathbf{e}}, 70^{\mathbf{e}}$ et $365^{\mathbf{e}}$ jours postpartum (dpp). 


\section{Résultats}

- Les néoplasies constituent la principale cause de décès des chiens irradiés durant la période prénatale.

- Parmi les néoplasies, les affections hématologiques dominent. Les lymphomes malins et désordres myéloprolifératifs représentent les néoplasies les plus fréquemment responsables de la mort des chiens beagles irradiés ( $25 \%$ des affections létales). Ces troubles hématologiques sont rares chez les témoins. Ils ont été décelés avec une particulière fréquence chez les chiens irradiés en fin de gestation.

- Après les problèmes hématologiques, les néoplasies les plus fréquemment déplorées sont celles touchant le sein, puis le poumon et la thyroïde. Enfin, des cancers de la peau, des surrénales et des testicules ont été observés en excès.

- Les chiens exposés durant la période périnatale (55 dpc et $2 \mathrm{dpp}$ ) sont victimes du plus grand nombre de cancers observés, et ce, tout particulièrement, durant le jeune âge.

- Les chiens irradiés à la fin de la période fœtale ont un risque accru de développer un cancer mortel durant la vie adulte.

- Les jeunes chiots irradiés font préférentiellement des cancers de la thyroïde.

Ces données suggèrent que l'âge au moment de l'exposition est déterminant vis-à-vis de la cancérogénèse radioinduite. Par ailleurs, la période fœtale terminale constitue, sans doute, le moment de la plus forte sensibilité.

C'est donc peu avant et peu après la naissance que le chien beagle est particulièrement sujet à la radiocancérogénèse.

\section{Epidémiologie humaine}

Plusieurs enquêtes épidémiologiques se sont intéressées au problème des cancers radioinduits après une irradiation prénatale. Parmi elles, retenons celle qui concerne les survivants japonais d'Hiroshima et de Nagasaki. Elle peut être facilement résumée en disant que l'on n'a observé aucun effet de ce type chez les sujets irradiés in utero jusqu'à présent.

Une autre enquête, par contre, mérite quelques développements. II s'agit, en effet, de travaux d'A. STEWART. Cet auteur a attiré l'attention sur l'effet cancérogène et tout spécialement leucémogène de l'irradiation in utero liée aux examens pelvimétriques pratiqués avant l'accouchement. Les doses reçues par le fœtus lors de tels examens étaient, il y a quelques années, d'environ 1 à 3 cGy, alors qu'actuellement, elles sont beaucoup plus faibles, puisqu'on les estime entre 0,5 et 1 cGy. A. STEWART a étudié les antécédents d'enfants atteints de leucémie et a trouvé, chez eux, une grande proportion d'irradiés in utero, supérieure de façon statistiquement significative à celle observée, en moyenne, chez des enfants non leucémiques du même sexe et du même âge. De tels résultats doivent être interprétés avec beaucoup de prudence pour les raisons suivantes :

- les courbes dose-réponse données par STEWART sont en désaccord avec les données d'Hiroshima et de Nagasaki ; 
- les mères des enfants leucémiques ont répondu de façon plus complète aux questions posées a posteriori que les mères d'enfants sains.

Cependant, ces explications ne permettent pas de nier formellement l'effet cancérogène des rayonnements ionisants administrés en fin de grossesse. La meilleure réponse à cette critique a été apportée par MOLE [16] qui a repris les données d'A. STEWART concernant les jumeaux, et son étude confirme, dans une certaine mesure, les résultats précités. II s'agit de la comparaison de l'incidence des cancers survenus chez des enfants uniques non irradiés durant la vie fœtale et chez les jumeaux dont les mères ont subi, dans un pourcentage non négligeable de cas, une pelvimétrie. Aucune pathologie particulière n'était à déplorer dans ce second groupe, si ce n'est... la gemellité. C'est ainsi que, seuls, $10 \%$ des enfants nés uniques avaient fait l'objet d'une pelvimétrie, alors que $50 \%$ des jumeaux en ont eu une. Or, bien que la fréquence des leucémies dans les deux groupes soit différente en l'absence d'irradiation in utero, l'excès de cancers et de leucémies observé chez les enfants irradiés in utero est le même chez les jumeaux et les enfants nés uniques. Ceci suggère la réalité de cet effet cancérogène. S'il existe, cet effet est très faible et ceci pourrait peut-être expliquer sa non-mise en évidence chez les sujets ayant été irradiés in utero lors des explosions d'Hiroshima et de Nagasaki.

TABLEAU ॥

\section{Effets cancérogènes d'une irradiation prénatale (pelvimétrie) d'après MOLE [17]}

\begin{tabular}{|c|c|c|c|c|}
\hline & & Pelvimétrie & Témoin & Excès \\
\hline Leucémies & {$\left[\begin{array}{c}\text { Enfant unique } \\
(10 \%) \\
\text { Jumeaux } \\
(50 \%)\end{array}\right.$} & $\begin{array}{l}3,4 \text { par } 10^{4} \\
2,6 \text { par } 10^{4}\end{array}$ & 2,3 par $10^{4}$ & $1,1.10^{-4}$ \\
\hline $\begin{array}{l}\text { Autres } \\
\text { cancers }\end{array}$ & $\begin{array}{l}\text { Enfant unique } \\
\text { Jumeaux }\end{array}$ & $\begin{array}{l}3,9 \text { par } 10^{4} \\
3,1 \text { par } 10^{4}\end{array}$ & $\begin{array}{l}2,7 \text { par } 10^{4} \\
2 \operatorname{par} 10^{4}\end{array}$ & $1,1 \cdot 10^{-4}$ \\
\hline
\end{tabular}

$\mathrm{Si}$, en se basant sur les résultats de cette étude anglaise, on compare l'effet leucémogène de cette irradiation in utero à celui observé chez l'adolescent ou chez l'adulte, on constate qu'il est 3 ou 4 fois plus grand. Ceci rejoint les constatations faites chez l'animal et relatées plus haut et peut s'expliquer par le fait que la mœlle du fœtus est en prolifération beaucoup plus active que celle de l'adolescent et devrait plutôt être comparée à une mœlle en régénération, laquelle est très radiosensible [37] ; en effet, un tissu est d'autant plus radiosensible que son cœfficient de prolifération est élevé. 
En pratique, il ne faut ni surestimer, ni nier ce genre de risque. L'augmentation liée à l'irradiation est, dans l'enquête, à peu près la même pour toutes les néoplasies et peu différente de 50 pour cent, ce qui paraît important, mais, étant donné que la fréquence des leucémies et des cancers chez l'enfant entre 0 et 15 ans est faible, de l'ordre de 1 pour 1000 , le nombre absolu de cancers et leucémies supplémentaires n'est pas très élevé : environ 0,5 pour 1000 pour une dose moyenne de 2 cGy. C'est peu avant la naissance et immédiatement après elle que la susceptibilité à la radiocancérogénèse est maximale. Ceci souligne, une fois de plus, que l'âge au moment de l'irradiation joue un rôle déterminant.

\section{CONCLUSION}

Bien que la sensibilité de l'embryon et du fœtus à l'irradiation semble exister durant toute la période de gestation à des degrés très variables, les travaux récents nous apprennent que l'effet dommageable principal est le retard mental.

II n'y a aucune donnée épidémiologique ou expérimentale qui permette de mettre en relation un effet tératogène quelconque avec une irradiation intervenue avant l'implantation.

Comme pour les autres effets des rayonnements, l'effet tératogène est diminué quand le débit est plus faible ou la dose fractionnée. Cependant, comme la radiosensibilité varie de façon très importante durant l'organogénèse, le fractionnement diminue la fréquence d'une anomalie particulière, mais tend à augmenter le nombre total de lésions.

Au plan pratique, il paraît essentiel de ne faire d'examens radiographiques chez une femme enceinte de 3 à 16 semaines, que si l'indication est impérative et ceci d'autant plus que des techniques nouvelles, telles que l'échographie, rendent souvent les mêmes services. Inversement, si une femme enceinte a subi un examen radiologique, il importe d'évaluer la dose reçue par l'embryon. Des experts pensent que si cette dose est inférieure à 10 cGy (10 rads), aucune mesure particulière ne s'impose, si ce n'est de prévenir efficacement toute autre irradiation. Ils conseillent l'avortement si la dose reçue par l'embryon est supérieure à 20 cGy (20 rads), mais rares sont les examens de radiodiagnostic qui délivrent de telles doses. Entre 10 et 20 cGy, chaque cas doit être discuté en fonction du contexte clinique et familial.

II ne s'agit là que de valeurs ayant pour seul but de fixer un ordre de grandeur.

Nous avons vu, en effet, que des doses de l'ordre de 10 cGy sont susceptibles de provoquer une résorption de l'œuf avant la nidation. Par ailleurs, en fin de grossesse et d'après certains auteurs, des doses de l'ordre de quelques centigrays, telles que celles délivrées au cours d'un examen pelvimétrique, seraient susceptibles d'entraîner une pathologie néoplasique excédentaire dans l'enfance.

II importe de signaler que certains auteurs (OFTEDAL, SCHULL) admettent l'hypothèse de la relation linéaire et sans seuil entre la dose et l'effet. 
Dans ce cas, la mort de toute cellule majore le risque de malformation. Cette hypothèse simplificatrice est, cependant, loin de faire l'unanimité.

Rappelons que la Commission internationale de protection radiologique a recommandé depuis longtemps d'éviter toute irradiation du petit bassin pendant les 15 jours précédant les règles, période pendant laquelle une grossesse insoupçonnée peut exister.

\section{BIBLIOGRAPHIE}

[1] AUXIER J.A. Radiation dosimetry for the survivors of the bombings of Hiroshima and Nagasaki. Oak Ridge National Laboratory report, 1977.

[2] BENJAMIN A. Health effects of prenatal and postnatal exposure to ionizing radiation in Beagle dogs. US Department of Health and Human Services, Radiological Health Laboratory. Annual Report, 1980. HHS-Publication FDA 82-8042, 1982.

[3] BENJAMIN S.A. et coll. Neoplasia in Beagle dogs given whole-body irradiation during prenatal or postnatal development. Fort Collins, Colorado State University, 1982.

[4] BRILL B., NANCE W. Radiation-induced abnormalities in human development. In "Advances in obstetrics and gynecology" (MARCUS S.L., Ed.) Baltimore : Williams et Wilkins, 1977, vol. 1, 69-87.

[5] BUCHET R. Gestation et radiodiagnostic. Contraintes légales du problème. Concours médical, 1982, 104, 1475-1483.

[6] Committee on the biological effects of ionizing radiations. National Academy of Sciences. National Research Council, Washington. The effects on populations of exposure to low levels of ionizing radiation, BEIR III. Washington : National academy of sciences, 1980.

[7] DOBBING J., SANDS J. The quantitative growth and development of the human brain. Arch. Dis. Childh., 1973 48, 757 à 767.

[8] DOBBING J. The pathogenesis of microcephaly with mental retardation. EULEP Symposium, Bordeaux, July 1982.

[9] FUCHS R. Low level radiation and infant mortality. Health Phys., 1981. 40, 847-854.

[10] GUSTAVSON K.H., JAGELL S., BLOMQUIST H.K., NORDENSON I. Microcephaly, mental retardation and chromosomal aberrations in a girl following radiation therapy during late fetal life. Acta Radiol. Oncol., 1981, 20, 209-212.

[11] HAAS J.F., SCHOTTENFELD D. Risks to the offspring from parental occupational exposures. J. occup. Med., 1979, 21, 607-613.

[12] HOFFMAN D. et coll. Effects of ionizing radiation on the developing embryo and fetus : a review. US Department of Health and Human Services, HHS Publication FDA 81-8170, 1981.

[13] HORNING S.J., HOPPE R.T., KAPLAN H.S., ROSENBERG S.A. Female reproductive potential after treatment for Hodgkin's disease. New Eng. J. Med., 1981. $23,1377-1382$.

[14] KALLINGER W., GRANNINGER W. The effect of a high-dose on a human foetus. Health Phys., 1979, 36, 1-6.

[15] MEYER M.B., TONASCIA J. Long-term effects of prenatal X-rays of human females. Amer. J. Epidemiol., 1981, 114, 304-336. 
[16] MOLE R.H. Antenatal irradiation and childhood cancer : causation or coïncidence. Br J. Cancer, 1974, 30, 199-208.

[17] MOLE R.H. Consequences of pre-natal radiation exposure for post-natal deveIopment, a review. Intern. J. Radiat. Biol., 1982, 42, 1-12.

[18] MOLE R.H. Human experience of radiation damage following prenatal exposure : its interpretation and implications. EULEP Symposium, Bordeaux, July 1982.

[19] MOLE R.H. Maldevelopment and malformation following intra uterine exposure to ionizing radiation. $11^{\mathrm{e}}$ Conférence de la Société Européenne de Tératologie, Paris, Août 1983.

[20] MOLLS M., STREFFER C., BEUNINGEN D., ZAMBOGLOU N. X-Irradiation in G2 phase of two-cell mouse embryos in vitro: cleavage, blastulation, cell kinetics and fetal development. Radiat. Res., 1982, 91, 219-234.

[21] MÜLLER W.U., STREFFER C. Combined effects after exposure during the preimplantation period. EULEP Symposium, Bordeaux, July 1982

[22] NATIONAL COUNCIL ON RADIATION PROTECTION AND MEASUREMENTS (NCRP). Review of NCRP radiation dose limit for embryo and fetus in occupationally exposed women. Washington : NCRP, 1977 (NCRP report 53).

[23] NATIONAL COUNCIL ON RADIATION PROTECTION AND MEASUREMENTS (NCRP). Medical radiation exposure of pregnant and potentially pregnant women. Washington : NCRP, 1977 (NCRP report 54).

[24] NEUMEISTER K. WASSER S. Findings in children after radiation exposure in utero from X-rays examinations of mothers. EULEP Symposium, Bordeaux 1982.

[25] NOMURA T. Parental exposure to $X$-rays and chemicals induces heritable tumours and anomalies in mice. Nature, 1982, 296,575-577.

[26] ORDY J.M., BRIZZEE K.R., DUNLAP W., KNIGHT C. Effects of prenatal ${ }^{60} \mathrm{Co}$ irradiation on postnatal neural learning, and hormonal development of the squirrel monkey. Radiat. Res., 1982, 89, 309-324.

[27] RON E. MODAN B., FLORO S., HARKEDAR I., GUREWITZ R. Mental function following scalp irradiation during childhood. Amer. J. Epidemiol., 1982, 116 , 149-160.

[28] RUGH R. X-ray induced teratogenesis in the mouse and its possible significance to man. Radiology, 1971, 99, 433-443.

[29] RUGH R. Does X-radiation of the preconceptional mammalian ovum lead to sterility and/or congenital anomalies? Fertility Sterility, 1975, 26, 560-572.

[30] RUGH R., MC MANAWAY M. Brain hernias in the mouse embryo as a consequence of low level prenatal X-ray exposure. In "Symposium on biological effects, imaging techniques, and dosimetry of ionizing radiations, June 6-8, 1979. US Department of Health and Human Services HHS - Publication (FDA) 80-8126, $1980,250-256$.

[31] SAMUEL J. Birth Defects research : 1980 and after. Amer. J. Med. 1982, 72, 119-126.

[32] SCHMAHL W. Long term effects after prenatal irradiation. EULEP Symposium, Bordeaux, July 1982.

[33] STORER J.B. Association between tumor types in irradiated BALB/C female mice. Radiat. Res., 1982, 92, 396-404.

[34] SYED I.B. SAMOLS E. Medical X-ray exposures of the human embryo and fetus. Health Phys., 1982, 42, 61-64.

[35] TEMPLIER J. PLANEL H. CHARPENTEAU M. MAUDUYT M.A. Effets de faibles doses de rayonnements ionisants sur le développement embryonnaire chez la souris. Bull. Assoc. Anat., 1981, 65, 341-350. 
[36] TOTTER J.R., MCPHERSON H.G. Do childhood cancers result from prenatal $X$-rays? Health Phys., 1981, 40, 511-524.

[37] TUBIANA M. Problemes posés par l'irradiation des femmes enceintes. Effets des radiations ionisantes sur l'embryon et le fœtus. Bull. Cancer, 1979, 66, 155-164.

[38] WALINDER G., RONNBACK C. Neoplastic effects after prenatal irradiation. EULEP Symposium, Bordeaux, 1982.

[39] WARREN S. Radiation damage in utero. In: Atomic medicine, Baltimore : Williams et Wilkins, 1969, 340-349.

[40] WEBSTER E. On the question of cancer induction by small $X$-ray doses. Amer. J. Roentgenol., 1981, 137, 647-666.

[41] WITHROW T. et coll. Possible damage from diagnostic X-irradiation. A review. US Department of Health and Human Services. HHS Publication FDA 80-8129, 1980. 